\title{
Non-integrability of the dumbbell and point mass problem
}

\author{
Andrzej J. Maciejewski • Maria Przybylska • \\ Leon Simpson • Wojciech Szumiński
}

Received: 22 April 2013 / Revised: 3 August 2013 / Accepted: 10 August 2013 /

Published online: 3 September 2013

(C) The Author(s) 2013. This article is published with open access at Springerlink.com

\begin{abstract}
This paper discusses a constrained gravitational three-body problem with two of the point masses separated by a massless inflexible rod to form a dumbbell. This problem is a simplification of a problem of a symmetric rigid body and a point mass, and has numerous applications in Celestial Mechanics and Astrodynamics. The non-integrability of this system is proven. This was achieved thanks to an analysis of variational equations along a certain particular solution and an investigation of their differential Galois group. Nowadays this approach is the most effective tool for study integrability of Hamiltonian and non-Hamiltonian systems.
\end{abstract}

Keywords Three-body problem · Morales-Ramis theory · Differential Galois theory · Non-integrability

\section{Equations of motion, symmetries and reduction}

Considered is the gravitational three-body problem with a single constraint. Three point masses, $m_{1}, m_{2}$ and $m_{3}$ move in a plane under mutual gravitational interaction. Masses $m_{2}$ and $m_{3}$ are connected by a massless inflexible rod of length $l>0$ to form a dumbbell. A pictorial description of the problem is given in Fig. 1. Various celestial objects are considered to possess such bimodal mass distribution because do not have enough gravitational force to form their shape into a spherical object, e.g. some asteroids such as (51) Nemausa and (216) Kleopatra; meteorites, especially large irons or nucleus of some comets e.g. Comet Borrelly (for detailed references see Povenmire 2002).The dumbbell satellite has attracted the attention of scientists since the middle of 20th century because it is suitable for an investigation of the general properties of the rigid body motion in a gravity field and provides important

\footnotetext{
A. J. Maciejewski $(\varangle) \cdot$ L. Simpson

Kepler Institute of Astronomy, University of Zielona Góra, Licealna 9, 65-407 Zielona Góra, Poland e-mail: andrzej.j.maciejewski@gmail.com 
Fig. 1 A dumbbell and a point mass in the plane. Point $O$ denotes the origin of an inertial frame. Point $C$ is the system centre of masses. Axis $O X_{1}$ of the moving frame is always parallel to the dumbbell

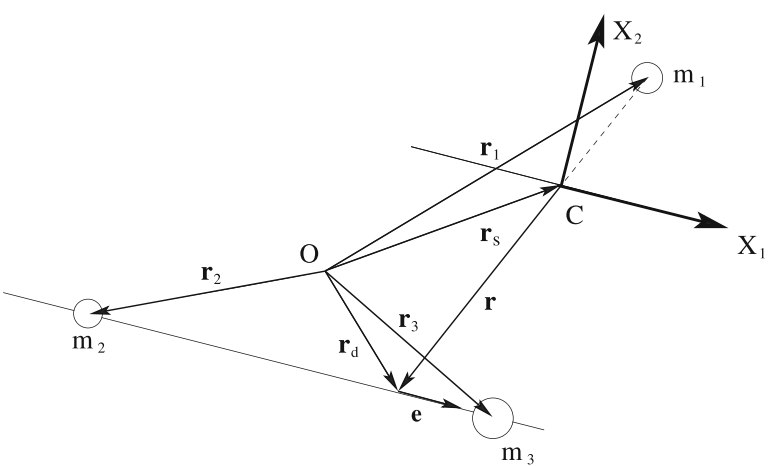

features for tethered satellite systems. See section 5.9 in Celletti (2010) and some original articles and references therein (Celletti and Sidorenko 2008; Guirao et al. 2013; Khan and Goel 2011; Schechter 1964; Vera 2013). Moreover dumbbell can be considered as a simplified model of a large spacecraft or an orbital cable system equipped with an elevator (see e.g. Burov et al. 2012; Okunev 1969; Sanyal et al. 2005). In most of the above examples the restricted version of the problem is considered. That is the mass center of a dumbbell moves on a given orbit and its rotational motion does not disturb the orbital motion. In many cases, e.g., for some double asteroids, such approximation is not well justified. This is why we consider unrestricted problem. An unrestricted problem of a point and axially symmetric body whose gravity field is approximated by a dumbbell field is considered in Goździewski and Maciejewski (1999).

The Lagrangian of the system has the form

$$
L=\frac{1}{2} m_{1}\left\|\dot{\mathbf{r}}_{1}\right\|^{2}+\frac{1}{2} m_{2}\left\|\dot{\mathbf{r}}_{2}\right\|^{2}+\frac{1}{2} m_{3}\left\|\dot{\mathbf{r}}_{3}\right\|^{2}-U\left(\mathbf{r}_{1}, \mathbf{r}_{2}, \mathbf{r}_{3}\right),
$$

where $\mathbf{r}_{1}, \mathbf{r}_{2}$ and $\mathbf{r}_{3}$ are the position vectors of the respective masses, and

$$
U\left(\mathbf{r}_{1}, \mathbf{r}_{2}, \mathbf{r}_{3}\right)=-\frac{G m_{1} m_{2}}{\left\|\mathbf{r}_{1}-\mathbf{r}_{2}\right\|}-\frac{G m_{1} m_{3}}{\left\|\mathbf{r}_{1}-\mathbf{r}_{3}\right\|}-\frac{G m_{2} m_{3}}{\left\|\mathbf{r}_{2}-\mathbf{r}_{3}\right\|},
$$

is the potential. Let $\mathbf{r}_{\mathrm{d}}$ is the radius vector of the centre of mass of the dumbbell, and

$$
\mathbf{e}:=\frac{\mathbf{r}_{3}-\mathbf{r}_{2}}{\left\|\mathbf{r}_{3}-\mathbf{r}_{2}\right\|}
$$

is a unit vector along the dumbbell. It follows that

$$
\begin{array}{ll}
\mathbf{r}_{2}=\mathbf{r}_{\mathrm{d}}+\mu_{3} l \mathbf{e}, & \dot{\mathbf{r}}_{2}=\dot{\mathbf{r}}_{\mathrm{d}}+\mu_{3} l \dot{\mathbf{e}}, \\
\mathbf{r}_{3}=\mathbf{r}_{\mathrm{d}}-\mu_{2} l \mathbf{e}, & \dot{\mathbf{r}}_{3}=\dot{\mathbf{r}}_{\mathrm{d}}-\mu_{2} l \dot{\mathbf{e}},
\end{array}
$$

where

$$
\mu_{2}:=\frac{m_{2}}{m_{\mathrm{d}}}, \quad \mu_{3}:=\frac{m_{3}}{m_{\mathrm{d}}}, \quad m_{\mathrm{d}}:=m_{2}+m_{3} .
$$

The system has five degrees of freedom. The configuration space of the point $m_{1}$ is $\mathbb{R}^{2}$, and the configuration space of the dumbbell is $\mathbb{R}^{2} \times \mathbb{S}^{1}$. A configuration of the system is fully specified by $\mathbf{r}_{1}, \mathbf{r}_{\mathrm{d}}$, and $\mathbf{e}$. In these coordinates, the Lagrangian is as follows:

$$
L=\frac{1}{2} m_{1}\left\|\dot{\mathbf{r}}_{1}\right\|^{2}+\frac{1}{2} m_{\mathrm{d}}\left\|\dot{\mathbf{r}}_{\mathrm{d}}\right\|^{2}+\frac{1}{2} I\|\dot{\mathbf{e}}\|^{2}-\widetilde{U}\left(\mathbf{r}_{1}, \mathbf{r}_{\mathrm{d}}, \mathbf{e}\right),
$$


where

$$
I:=l^{2} \frac{m_{2} m_{3}}{m_{\mathrm{d}}}, \quad \widetilde{U}\left(\mathbf{r}_{1}, \mathbf{r}_{\mathrm{d}}, \mathbf{e}\right)=U\left(\mathbf{r}_{1}, \mathbf{r}_{\mathrm{d}}+\mu_{3} l \mathbf{e}, \mathbf{r}_{\mathrm{d}}-\mu_{2} l \mathbf{e}\right) .
$$

The system possesses natural symmetries that are exploited to reduce its dimension. Amongst these is translational symmetry. This is manifest by defining $\mathbf{r}:=\mathbf{r}_{\mathrm{d}}-\mathbf{r}_{1}$ as the vector between the mass $m_{1}$ and the dumbbell's centre of mass. A configuration of the system can be thus described by $\mathbf{r}$, e, and $\mathbf{r}_{\mathrm{s}}$ defined by

$$
m_{\mathrm{s}} \mathbf{r}_{\mathrm{s}}=m_{1} \mathbf{r}_{1}+m_{\mathrm{d}} \mathbf{r}_{\mathrm{d}}, \quad m_{\mathrm{s}}:=m_{1}+m_{2}+m_{3} .
$$

As

$$
\mathbf{r}_{1}=\mathbf{r}_{\mathrm{s}}-\frac{m_{\mathrm{d}}}{m_{\mathrm{s}}} \mathbf{r}, \quad \mathbf{r}_{\mathrm{d}}=\mathbf{r}_{\mathrm{s}}+\frac{m_{1}}{m_{\mathrm{s}}} \mathbf{r}
$$

the Lagrangian (1.7) is written in the following form:

$$
L=\frac{1}{2} m_{\mathrm{r}}\|\dot{\mathbf{r}}\|^{2}+\frac{1}{2} I\|\dot{\mathbf{e}}\|^{2}+\frac{1}{2} m_{\mathrm{s}}\left\|\dot{\mathbf{r}}_{\mathrm{s}}\right\|^{2}-W(\mathbf{r}, \mathbf{e}),
$$

where

$$
m_{\mathrm{r}}:=\frac{m_{1} m_{\mathrm{d}}}{m_{\mathrm{s}}}
$$

is the reduced mass, and

$$
W(\mathbf{r}, \mathbf{e})=-\frac{G m_{1} m_{2}}{\left\|\mathbf{r}+\mu_{3} l \mathbf{e}\right\|}-\frac{G m_{1} m_{3}}{\left\|\mathbf{r}-\mu_{2} l \mathbf{e}\right\|} .
$$

The components of $\mathbf{r}_{\mathrm{s}}$ are cyclic coordinates, and the motion of the centre of mass separates completely. Therefore, the term $m_{\mathrm{s}}\left\|\dot{\mathbf{r}}_{\mathrm{s}}\right\|^{2} / 2$ is removed from the Lagrangian (1.11).

It is convenient to introduce dimensionless variables, taking $l$ as the unit of length, $m_{\mathrm{r}}$ as the unit of mass, and

$$
T:=\sqrt{\frac{l^{3}}{m_{\mathrm{s}}}},
$$

as the unit of time. Setting $l=m_{\mathrm{r}}=T=1$, the Lagrangian (1.11) reduces to

$$
L=\frac{1}{2}\|\dot{\mathbf{r}}\|^{2}+\frac{1}{2} I\|\dot{\mathbf{e}}\|^{2}-W(\mathbf{r}, \mathbf{e}),
$$

where

$$
W(\mathbf{r}, \mathbf{e})=-\frac{1}{\|(1+\mu) \mathbf{r}+\mu \mathbf{e}\|}-\frac{\mu}{\|(1+\mu) \mathbf{r}-\mathbf{e}\|}, \quad \mu:=\frac{m_{3}}{m_{2}} .
$$

The reduced system still has a symmetry: it is invariant with respect to the natural action of the group $\mathrm{SO}(2, \mathbb{R})$. This symmetry can be used to reduce the dimension of the configuration space by one. This is achieved by describing the dynamics in a rotating frame in which the dumbbell is at rest. The transformation from the inertial frame to the rotating frame is given by

$$
\mathbf{r}=\mathbf{A R}, \quad \mathbf{A} \in \mathrm{SO}(2, \mathbb{R}) .
$$

An additional assumption, that $\mathbf{e}$ is parallel to $x$-axis of the rotating frame, allows for a coordinate representation of the transformation as follows:

$$
\mathbf{A}=\left[\begin{array}{cc}
\cos \varphi & -\sin \varphi \\
\sin \varphi & \cos \varphi
\end{array}\right], \quad \text { and } \quad \mathbf{e}=[\cos \varphi, \sin \varphi]^{T} .
$$


Then

$$
\mathbf{e}=\mathbf{A E}, \quad \mathbf{E}=[1,0]^{T},
$$

and

$$
\dot{\mathbf{e}}=\dot{\varphi}[-\sin \varphi, \cos \varphi]^{T}, \quad \dot{\mathbf{A}}=\dot{\varphi} \mathbf{A} \mathbf{J}, \quad \mathbf{J}=\left[\begin{array}{rr}
0 & -1 \\
1 & 0
\end{array}\right] .
$$

The Lagrangian expressed in the coordinates $\mathbf{R}=\left[X_{1}, X_{2}\right]^{T}$, and $\varphi$ has the form

$$
L=\frac{1}{2}\|\dot{\mathbf{R}}\|^{2}+\dot{\varphi} \dot{\mathbf{R}}^{T} \mathbf{J R}+\frac{1}{2} \dot{\varphi}^{2}\|\mathbf{R}\|^{2}+\frac{1}{2} I \dot{\varphi}^{2}-V(\mathbf{R})
$$

where

$$
V(\mathbf{R}):=-\frac{1}{\|(1+\mu) \mathbf{R}+\mu \mathbf{E}\|}-\frac{\mu}{\|(1+\mu) \mathbf{R}-\mathbf{E}\|} .
$$

The generalised momenta $\mathbf{P}:=\left[P_{1}, P_{2}\right]^{T}$ and $P_{\varphi}$, are given by

$$
\mathbf{P}:=\frac{\partial L}{\partial \dot{\mathbf{R}}}=\dot{\mathbf{R}}+\dot{\varphi} \mathbf{J R}, \quad P_{\varphi}:=\frac{\partial L}{\partial \dot{\varphi}}=\dot{\varphi}\left(\|\mathbf{R}\|^{2}+I\right)+\dot{\mathbf{R}}^{T} \mathbf{J R} .
$$

Coordinate $\varphi$ is cyclic, so $P_{\varphi}$ is a first integral of the system. Thus, the Hamiltonian of the system is written as

$$
H=\frac{1}{2}\|\mathbf{P}\|^{2}+\frac{1}{2} \alpha\left(\gamma-\mathbf{P}^{T} \mathbf{J R}\right)^{2}+V(\mathbf{R}),
$$

where $\alpha=1 / I$, and $\gamma$ is a fixed value of the first integral $P_{\varphi}$ corresponding to the cyclic variable $\varphi$. Hence, the equations of motion have the form

$$
\begin{aligned}
\frac{\mathrm{d}}{\mathrm{d} t} \mathbf{R}= & \mathbf{P}-\alpha\left(\gamma-\mathbf{P}^{T} \mathbf{J R}\right) \mathbf{J R}, \\
\frac{\mathrm{d}}{\mathrm{d} t} \mathbf{P}= & -\alpha\left(\gamma-\mathbf{P}^{T} \mathbf{J R}\right) \mathbf{J P}-(1+\mu) \frac{(1+\mu) \mathbf{R}+\mu \mathbf{E}}{\|(1+\mu) \mathbf{R}+\mu \mathbf{E}\|^{3}}- \\
& \mu(1+\mu) \frac{(1+\mu) \mathbf{R}-\mathbf{E}}{\|(1+\mu) \mathbf{R}-\mathbf{E}\|^{3}} .
\end{aligned}
$$

\section{Problem}

Numerical experiments suggest that the considered system is not integrable. The complex behaviour of the system is apparent from the Poincare cross-section. For fixed values of the parameters and the energy the equations (1.25) are integrated numerically by the BulirschStoer method (Press et al. 1992). The cross-section plane is specified as $X_{1}=0$, and the cross direction is chosen $\dot{X}_{1}>0$. The coordinates of the cross-section are $\left(X_{2}, P_{2}\right)$. The cross-sections presented in the subsequent figures are obtained for parameters $\mu=1$ and $\alpha=\frac{4}{61}$.

Figure 2 is a cross-section corresponding to energy $e=-0.1$ and $\gamma=-1$. Chaotic behaviour is evident. Two cross-sections for $\gamma=0$ are shown in Figs. 3 and 4. Both of them attest to the non-integrability of the system. Let us remark here that all cross sections were done for negative energy values. For non-negative values of energy the energy levels are non-compact. This is why trajectories in such levels usually do not return to the cross section plane. In the best case investigating Poincaré cross-section we observe that a single trajectory 
Fig. 2 Cross-section for energy $e=-0.1$ and $\gamma=-1$

Fig. 3 Cross-section for $e=-0.2$ and $\gamma=0$

Fig. 4 Cross-section for $e=-0.15$ and $\gamma=0$
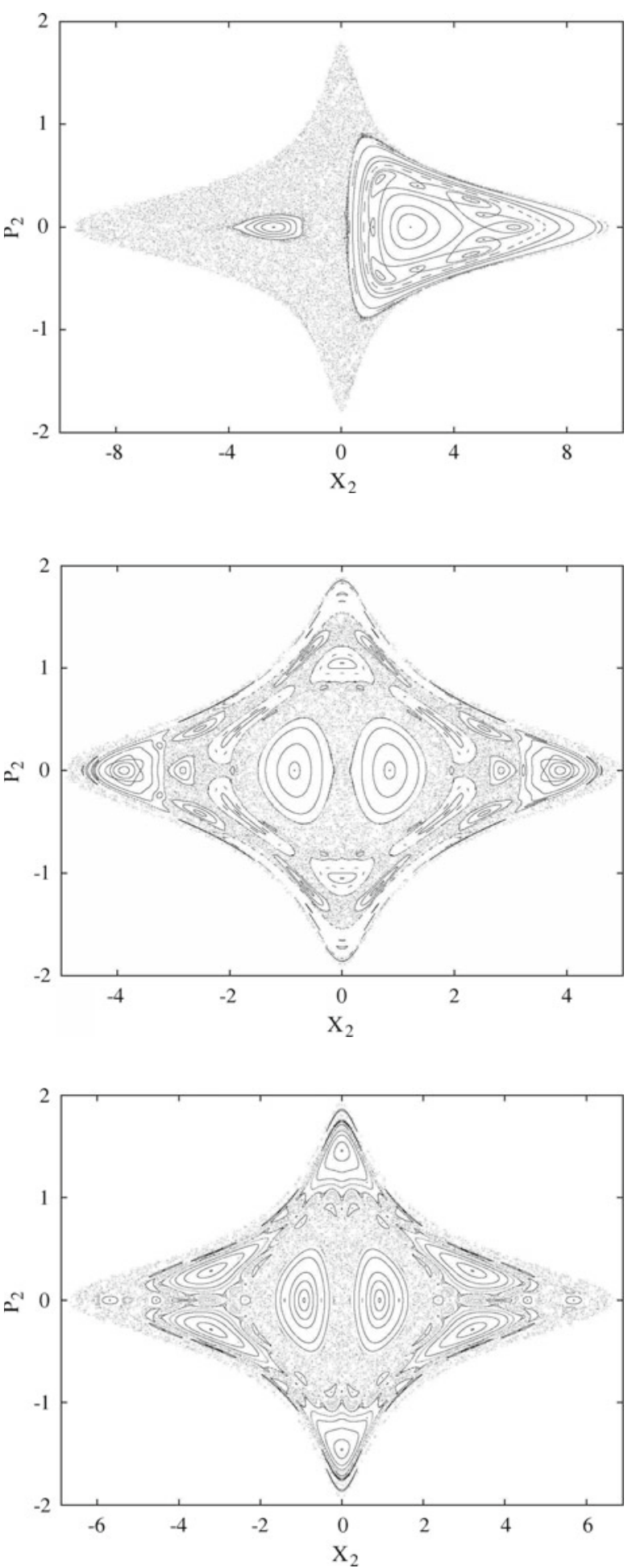
intersects the cross plane along a spiral. No useful information about global properties of the system can be deduced.

The problem is to prove the non-integrability of the system analytically. It is attractive to try using the differential Galois approach to the problem of integrability (see e.g. Morales Ruiz 1999). This method was applied successfully to many systems and in particular to various Celestial Mechanics problems such as e.g.: three body problem (Boucher and Weil 2003; Maciejewski and Przybylska 2011; see also Tsygvintsev 2001, 2003, 2007), satellite in geo-magnetic field (Boucher 2006; Maciejewski and Przybylska 2003), two-body problems in constant curvature spaces (Maciejewski and Przybylska 2003), some $n$-body problems (Combot 2012; Simon 2007; Tosel 1998, 1999), Sitnikov's three-body problem (Morales Ruiz 1999), Hill's problem (Morales-Ruiz et al. 2005), generalized two-fixed centres problem whose interaction potential is $V=a r^{2 n}$ (Maciejewski and Przybylska 2004), generalized anisotropic Kepler problem (Arribas et al. 2003) and many others. However, as is explained in (Combot 2013), application of this theory directly to the system (1.25) is invalid as the Hamiltonian function (1.24) is not single-valued.

Proposed in this paper is a solution addressing this deficiency, based on an extension of the system into a larger phase space. Coordinates of these additional dimensions are denoted by $R_{1}$ and $R_{2}$. This approach further requires a definition of the time derivative consistent with system (1.25). Following this, coordinates $R_{1}$ and $R_{2}$ are considered as lengths of vectors

$$
\mathbf{R}_{1}:=(1+\mu) \mathbf{R}+\mu \mathbf{E}, \quad \mathbf{R}_{2}:=(1+\mu) \mathbf{R}-\mathbf{E} .
$$

That is, equations $G_{1}=G_{2}=0$, where polynomials $G_{1}, G_{2} \in \mathbb{C}\left[X_{1}, X_{2}, R_{1}, R_{2}\right]$ given by

$$
\left.\begin{array}{l}
\left.G_{1}:=R_{1}^{2}-\left[(1+\mu) X_{1}+\mu\right)\right]^{2}-(1+\mu)^{2} X_{2}^{2}=R_{1}^{2}-\mathbf{R}_{1}^{T} \mathbf{R}_{1}, \\
\left.G_{2}:=R_{2}^{2}-\left[(1+\mu) X_{1}-1\right)\right]^{2}-(1+\mu)^{2} X_{2}^{2}=R_{2}^{2}-\mathbf{R}_{2}^{T} \mathbf{R}_{2} .
\end{array}\right\}
$$

define $R_{1}$ and $R_{2}$ as algebraic functions of $\left(X_{1}, X_{2}\right)$.

The extended phase space is $\mathbb{C}^{6}$ with coordinates $\mathbf{Z}:=\left[X_{1}, X_{2}, P_{1}, P_{2}, R_{1}, R_{2}\right]$. The Hamiltonian (1.24) expressed in these coordinates reads

$$
K=K(\mathbf{Z}):=\frac{1}{2}\|\mathbf{P}\|^{2}+\frac{1}{2} \alpha\left(\gamma-\mathbf{P}^{T} \mathbf{J R}\right)^{2}-\frac{1}{R_{1}}-\frac{\mu}{R_{2}} .
$$

As

$$
\frac{\mathrm{d}}{\mathrm{d} t} R_{i}=\frac{\mathbf{R}_{i}^{T} \dot{\mathbf{R}}_{i}}{R_{i}}=(1+\mu) \frac{\mathbf{R}_{i}^{T} \dot{\mathbf{R}}}{R_{i}}, \quad i=1,2,
$$

system (1.25) can be extended to the following one.

$$
\frac{\mathrm{d}}{\mathrm{d} t} \mathbf{Z}=\mathbf{U}(\mathbf{Z}), \quad \mathbf{U}(\mathbf{Z}):=\mathbf{J}(\mathbf{Z}) K^{\prime}(\mathbf{Z}), \quad \mathbf{J}(\mathbf{Z}):=\left[\begin{array}{rll}
\mathbf{0}_{2} & \mathbf{E}_{2} & \mathbf{0}_{2} \\
-\mathbf{E}_{2} & \mathbf{0}_{2} & -\mathbf{A} \\
\mathbf{0}_{2} & \mathbf{A}^{T} & \mathbf{0}_{2}
\end{array}\right],
$$

where

$$
\mathbf{A}=(1+\mu)\left[\frac{\mathbf{R}_{1}}{R_{1}}, \frac{\mathbf{R}_{2}}{R_{2}}\right], \quad K^{\prime}(\mathbf{Z}):=\left(\nabla_{\mathbf{Z}} K(\mathbf{Z})\right)^{T} .
$$

It can be shown that matrix $\mathbf{J}(\mathbf{Z})$ defines a Poisson structure on $\mathbb{C}^{6}$. The corresponding Poisson bracket is given by the following formula

$$
\{F, G\}(\mathbf{Z})=F^{\prime}(\mathbf{Z})^{T} \mathbf{J}(\mathbf{Z}) G^{\prime}(\mathbf{Z}),
$$


where $F$ and $G$ are two smooth functions. The Poisson structure given by $\mathbf{J}(\mathbf{Z})$ is degenerated and has rank 4. It is demonstrable that polynomials $G_{1}$ and $G_{2}$ are Casimir functions of this structure, whose common levels are symplectic manifolds. In particular,

$$
M_{\mu}^{4}:=\left\{\mathbf{Z} \in \mathbb{C}^{6} \mid G_{1}(\mathbf{Z})=G_{2}(\mathbf{Z})=0\right\}, \quad \mu \in(0,1 / 2] .
$$

is a symplectic manifold. System (2.5), when restricted to $M_{\mu}^{4}$, is equivalent to (1.25).

In the setting thus described the main result of this paper can be formulated in the following theorem.

Theorem 2.1 For $\gamma=0$ and $\mu \in(0,1 / 2]$, system (2.5), when restricted to the symplectic leaf $M_{\mu}^{4}$, is not integrable in the Liouville sense with meromorphic first integrals.

\section{Particular solution and variational equation}

If $\gamma=0$, then Eq. (2.5) have an invariant algebraic manifold,

$$
\mathcal{N}:=\left\{\mathbf{Z} \in \mathbb{C}^{6} \mid X_{2}=P_{2}=0, \quad G_{1}(\mathbf{Z})=G_{2}(\mathbf{Z})=0\right\} .
$$

On this manifold it can be taken that

$$
R_{1}=(1+\mu) X_{1}+\mu, \quad R_{2}=(1+\mu) X_{1}-1,
$$

so the system (2.5), restricted to $\mathcal{N}$, reduces to

$$
\dot{X}=P, \quad \dot{P}=-(\mu+1)\left(\frac{\mu}{[(1+\mu) X-1]^{2}}+\frac{1}{[(1+\mu) X+\mu]^{2}}\right),
$$

where $(X, P):=\left(X_{1}, P_{1}\right) \in \mathbb{C}^{2}$. This is a Hamiltonian system with one degree of freedom. The point and the dumbbell move in one line. Fixing the energy $e$ we have a particular solution $(X(t), P(t))$. Thus the manifold $\mathcal{N}$ is foliated by phase curves $\Gamma_{e}$ on energy levels $K_{\mid \mathcal{N}}=e$, that is

$$
\Gamma_{e} \subset M_{e}:=\left\{(X, P) \in \mathbb{C}^{2} \mid e=\frac{1}{2} P^{2}-\frac{\mu}{(1+\mu) X-1}-\frac{1}{(1+\mu) X+\mu}\right\} .
$$

These curves are give parametrically $t \longmapsto(X(t), P(t))$ For a generic value of $e$ the level $M_{e}$ contains three phase curves. If $e$ is real, two or three of these levels have a non-empty intersection with the real part of the phase space. Depending on the real value of $e$, the point can move either between the end masses of the dumbbell or outside them. In further consideration it is assumed that $e \geq 0$, and the chosen phase curve $\Gamma_{e}$ contains the halfline $(1 /(1+\mu), \infty) \times\{0\} \subset \mathbb{C}^{2}$. The other choices are equally good for the proof of non-integrability. We made such a choice because it describes real, physically meaningful solution. In the phase space $\mathbb{C}^{6}$ this curve is given by

$$
\boldsymbol{\Gamma}_{e}:=\left\{\mathbf{Z} \in \mathcal{N} \mid\left(X_{1}, P_{1}\right) \in \Gamma_{e}\right\} .
$$

Application of the Morales-Ramis theory necessitates the linearisation of Eq. (2.5) along $\Gamma_{e}$. It has the form

$$
\frac{\mathrm{d}}{\mathrm{d} t} \mathbf{z}=\mathbf{V} \mathbf{z}, \quad \mathbf{V}=\frac{\partial \mathbf{U}}{\partial \mathbf{Z}}(\mathbf{Z}), \quad \mathbf{Z} \in \boldsymbol{\Gamma}_{e} .
$$

This system has three first integrals

$$
k(\mathbf{z}):=\nabla K(\mathbf{Z}) \cdot \mathbf{z}, \quad g_{1}(\mathbf{z}):=\nabla G_{1}(\mathbf{Z}) \cdot \mathbf{z}, \quad g_{2}(\mathbf{z}):=\nabla G_{2}(\mathbf{Z}) \cdot \mathbf{z}, \quad \mathbf{Z} \in \boldsymbol{\Gamma}_{e} .
$$


On the level $g_{1}(\mathbf{z})=g_{2}(\mathbf{z})=0$, the following equalities are satisfied

$$
R_{1} r_{1}=(1+\mu)\left[(1+\mu) X_{1}+\mu\right] x_{1}, \quad R_{2} r_{2}=(1+\mu)\left[(1+\mu) X_{1}-1\right] x_{1},
$$

where it is assumed that $\mathbf{z}=\left[x_{1}, x_{2}, p_{1}, p_{2}, r_{1}, r_{2}\right]^{T}$. On $\boldsymbol{\Gamma}_{e}$, coordinates $\left(R_{1}, R_{2}\right)$ can be expressed by $X_{1}$,

$$
R_{1}=(1+\mu) X_{1}+\mu, \quad R_{2}=(1+\mu) X_{1}-1 .
$$

Hence, on the level $g_{1}(\mathbf{z})=g_{2}(\mathbf{z})=0$, variables $\left(r_{1}, r_{2}\right)$ can be eliminated from the variational equations (3.6). The obtained reduced system of variational equations has the form

$$
\frac{\mathrm{d}}{\mathrm{d} t}\left[\begin{array}{l}
u_{1} \\
v_{1} \\
u_{2} \\
v_{2}
\end{array}\right]=\left[\begin{array}{llll}
0 & 1 & 0 & 0 \\
A & 0 & 0 & 0 \\
0 & 0 & -\alpha X P & 1+\alpha X^{2} \\
0 & 0 & B & \alpha X P
\end{array}\right]\left[\begin{array}{l}
u_{1} \\
v_{1} \\
u_{2} \\
v_{2}
\end{array}\right],
$$

where

$$
\begin{aligned}
& A=2(\mu+1)^{2}\left(\frac{\mu}{[(1+\mu) X-1]^{3}}+\frac{1}{[(1+\mu) X+\mu]^{3}}\right), \\
& B=-\alpha P^{2}-\frac{1}{2} A,
\end{aligned}
$$

and $(X, P) \in \Gamma_{e}$. This system splits into two subsystems. Variables $\left(u_{1}, v_{1}\right)$ describe variations in the invariant plane $\mathcal{N}$. In fact, $\left(u_{1}, v_{1}\right)$ is a vector tangent to $\mathcal{N}$ at point $(X, P)$, hence the subsystem in variables $\left(u_{1}, v_{1}\right)$ is called tangential. The second subsystem, corresponding to variables $\left(u_{2}, v_{2}\right)$, yields the normal variational equations. The latter subsystem can be expressed by a single second-order equation,

$$
\ddot{u}+a \dot{u}+b u=0,
$$

where $u=u_{2}$, and

$$
a:=-\frac{2 \alpha X P}{1+\alpha X^{2}}, \quad b:=\frac{2 \alpha P^{2}}{1+\alpha X^{2}}+\frac{1}{2}\left(1+\alpha X^{2}\right) A .
$$

Changing the independent variable by

$$
t \longrightarrow z=(1+\mu) X(t)
$$

transforms equation (3.12) into an equation with rational coefficients. This follows from

$$
\begin{aligned}
\frac{\mathrm{d}}{\mathrm{d} t} & =\dot{z} \frac{\mathrm{d}}{\mathrm{d} z}, \quad \frac{\mathrm{d}^{2}}{\mathrm{~d} t^{2}}=(\dot{z})^{2} \frac{\mathrm{d}^{2}}{\mathrm{~d} z^{2}}+\ddot{z} \frac{\mathrm{d}}{\mathrm{d} z}, \\
\dot{z} & =(1+\mu) \dot{x}=(1+\mu) p_{1}, \quad(\dot{z})^{2}=(1+\mu)^{2} p_{1}^{2}=2(1+\mu)^{2}\left(e+\frac{\mu}{z-1}+\frac{1}{z+\mu}\right), \\
\ddot{z} & =(1+\mu) \ddot{p}_{1}=-(\mu+1)^{2}\left(\frac{\mu}{(z-1)^{2}}+\frac{1}{(z+\mu)^{2}}\right),
\end{aligned}
$$

where the energy integral

$$
e=\frac{1}{2} \dot{X}^{2}-\frac{\mu}{(1+\mu) X-1}-\frac{1}{(1+\mu) X+\mu}
$$

has been used. 
Resultantly, Eq. (3.12) transforms into

$$
u^{\prime \prime}+p(z) u^{\prime}+q(z) u=0
$$

where the prime denotes the derivative with respect to $z$, and

$$
\begin{aligned}
p= & -\left[(-1+z)^{2}\left(1+z^{2}(5+4 e z) \alpha\right)+\left(2+z\left(-4+3 z+\left(4+z\left(-8+8 e(-1+z)^{2}\right.\right.\right.\right.\right. \\
& \left.\left.\left.\left.+5 z^{2}\right)\right) \alpha\right)\right) \mu+\left(1+3 z^{2}+2 z\left(2 e(-1+z)^{2}+z(-4+5 z)\right) \alpha\right) \mu^{2}+(1+z(4+z-4 \alpha \\
& \left.+5 z \alpha)) \mu^{3}+2(1+z) \mu^{4}+\mu^{5}\right] /[2(z-1)(z+\mu)((z-1)(1+e z)+(e(z-1)+z) \mu \\
& \left.\left.+\mu^{2}\right)\left(z^{2} \alpha+(1+\mu)^{2}\right)\right], \\
q= & {\left[4(z-1)^{2} \alpha(1+\mu)(z+\mu)^{2}\left((z-1)(1+e z)+(e(z-1)+z) \mu+\mu^{2}\right)+\left((z-1)^{3}\right.\right.} \\
& \left.\left.+(1+3(z-1) z) \mu+(3 z-1) \mu^{2}+\mu^{3}\right)\left(z^{2} \alpha+(1+\mu)^{2}\right)^{2}\right] /\left[2(z-1)^{2}(1+\mu)\right. \\
& \left.\cdot(z+\mu)^{2}\left((z-1)(1+e z)+(e(z-1)+z) \mu+\mu^{2}\right)\left(z^{2} \alpha+(1+\mu)^{2}\right)\right] .
\end{aligned}
$$

Making the next change of variables

$$
u=w \exp \left[-\frac{1}{2} \int_{z_{0}}^{z} p(s) d s\right],
$$

(3.14) is transformed to the canonical form

$$
w^{\prime \prime}=r(z) w, \quad r(z)=-q(z)+\frac{1}{2} p^{\prime}(z)+\frac{1}{4} p(z)^{2},
$$

with coefficients given as

$$
\begin{aligned}
r & =\frac{P}{M} \\
M & =16(z-1)^{2} \beta^{2}\left(z^{2}+\beta^{2}\right)^{2}(z+\mu)^{2}\left[(z-1)(1+e z)+(e(-1+z)+z) \mu+\mu^{2}\right]^{2}, \\
P & =\sum_{i=0}^{11} p_{i} z^{11-i} .
\end{aligned}
$$

Here $\beta^{2}=(1+\mu)^{2} / \alpha$ and coefficients $p_{i}$ of $P$ are given in the Appendix A. This equation has seven singularities

$$
\begin{aligned}
z_{1} & =0, \quad z_{2}=-\mu, \quad z_{3,4}= \pm \mathrm{i} \beta \\
z_{5,6} & =\frac{e-1-(1+e) \mu \pm \sqrt{(1+\mu)\left((1+e)^{2}+(e-1)^{2} \mu\right)}}{2 e},
\end{aligned}
$$

and $z_{7}=\infty$, providing energy is chosen such that

$$
\begin{aligned}
e \notin\{ & \frac{(1 \pm \mathrm{i} \beta)( \pm \mathrm{i}+\beta \mp \mathrm{i} \mu)(\beta \pm \mathrm{i} \mu)(1+\mu)}{\left(1+\beta^{2}\right)\left(\beta^{2}+\mu^{2}\right)}, \frac{(\sqrt{\mu} \pm \mathrm{i})^{2}}{\mu+1}, \\
& \left.\frac{-\mu+\mu^{3} \pm \mathrm{i}\left(1+\mu^{3}+\beta^{2}(1+\mu)\right)|\beta|}{\left(1+\beta^{2}\right)\left(\beta^{2}+\mu^{2}\right)}\right\}
\end{aligned}
$$

Points $z_{1}, \ldots, z_{6}$ are regular poles of second order and the order of infinity is 1 provided that $E(1+\mu) \neq 0$. Here, the order of a singular point is defined as in (Kovacic 1986), see also Appendix B. 
The differences of exponents at the singularities in $\mathbb{C}$ are as follows

$$
\Delta_{1}=\frac{1}{2} \sqrt{1-\frac{8}{\beta^{2}}}, \quad \Delta_{2}=\frac{1}{2} \sqrt{1-\frac{8 \mu^{2}}{\beta^{2}}}, \quad \Delta_{3}=\Delta_{4}=2, \quad \Delta_{5}=\Delta_{6}=\frac{1}{2} .
$$

It is evident that the differences of the exponents at singularities $z_{3}$ and $z_{4}$ are integer, and thus, in local solutions around these points, logarithmic terms may appear. Application of the method described in Appendix B verifies the presence of such terms. For singularity $z_{3}$, solutions of the indicial equation are $\alpha_{1}=3 / 2$ and $\alpha_{2}=-1 / 2$. The relevant one is $\alpha=\alpha_{1}=3 / 2$. The expansion of $r\left(z-z_{3}\right)^{2}$, according to (B.4), gives coefficients $r_{0}, r_{1}$, and $r_{2}$. Then $f_{1}, f_{2}$ and $g_{1}, g_{2}$ are determined from (B.6) and (B.11), respectively. Since $s=\alpha_{1}-\alpha_{2}=2$, the coefficient $g_{2}$, which multiplies the logarithm, must be found, and it is given as

$$
g_{2}=-\frac{\mathrm{i}(1+\mu)\left((\mathrm{i}+\beta)^{2}+\mu-2 \mathrm{i} \beta \mu-\mu^{2}\right)}{8 \beta(\mathrm{i}+\beta)(\beta-\mathrm{i} \mu)\left(1+e \beta^{2}+(e-\mu) \mu-\mathrm{i} \beta(1+e(-1+\mu)+\mu)\right)} .
$$

Examination of the real and imaginary part of $g_{2}$ yields the following conditions

$$
\begin{aligned}
& \beta(1+\mu)\left[1+\beta^{4}(1+\mu)+2 \beta^{2}(-1+\mu)\left(-1-2 e \mu+\mu^{2}\right)\right. \\
& \left.\quad+\mu\left(-1-2 e(-1+\mu)\left(1+\mu^{2}\right)+\mu(-1+\mu(-1+(-1+\mu) \mu))\right)\right]=0, \\
& (1+\mu)\left[\mu\left(1+3 \beta^{2}+(-1+\mu) \mu\right)\left(-1+\mu^{2}\right)+e\left(\beta^{6}+\mu^{2}\left(-1+\mu-\mu^{2}\right)\right.\right. \\
& \left.\left.\quad+\beta^{4}(2+\mu(-3+2 \mu))+\beta^{2}(1+\mu(-1+\mu(-1+(-1+\mu) \mu)))\right)\right]=0 .
\end{aligned}
$$

This system has two solutions satisfying $\mu>0, \beta^{2}>0$ and $e \in \mathbb{R}$

$$
\beta^{2}=-1+\mu-\mu^{2}, e=\frac{1+\mu}{1-\mu} \text { and } \beta^{2}=\mu=1 .
$$

The condition precluding a logarithm in a local solution around $z_{4}$ is $g_{2}^{*}=0$, where * denotes complex conjugation. The corresponding solutions are the same as those given previously.

In the first solution in (3.21), condition

$$
\beta^{2}=-1+\mu-\mu^{2}
$$

gives

$$
\frac{m_{0}}{m_{1}}=-\frac{\mu(\mu+1)}{\mu^{2}+1} \leq 0,
$$

with the only non-negative solution $m_{1}=0$. The second solution in (3.21) also yields $m_{1}=0$ only.

If conditions (3.21) are not satisfied, the two linearly-independent local solutions $w_{1}$ and $w_{2}$ of (3.16) in a neighbourhood of $z_{*}=z_{3}$ or $z_{*}=z_{4}$ have the following forms

$$
w_{1}(z)=\left(z-z_{*}\right)^{\alpha} f(z), \quad w_{2}(z)=w_{1}(z) \ln \left(z-z_{*}\right)+\left(z-z_{*}\right)^{\alpha-2} h(z),
$$

where $f(z)$ and $h(z)$ are holomorphic at $z_{*}$ and $f\left(z_{*}\right) \neq 0$. The local monodromy matrix, which corresponds to the continuation of the matrix of fundamental solutions along a small loop encircling $z_{*}$ counter-clockwise, gives rise to a triangular monodromy matrix,

$$
\left[\begin{array}{ll}
-1 & -2 \pi \mathrm{i} \\
0 & -1
\end{array}\right]
$$


for details, see Maciejewski and Przybylska (2002). A subgroup of $\operatorname{SL}(2, \mathbb{C})$ generated by a triangular non-diagonalizable matrix is not finite, and thus the differential Galois group is not finite either. Moreover, the differential Galois group $\mathcal{G}$ of this equation is not any subgroup of the dihedral group because such subgroups contain only diagonalizable matrices. Thus, $\mathcal{G}$ is either the full triangular group or $\operatorname{SL}(2, \mathbb{C})$. But since the order of infinity is one, the necessary condition that $\mathcal{G}$ is the full triangular group is not satisfied (see Lemma B.2). This means that the differential Galois group of $(3.16)$ is $\operatorname{SL}(2, \mathbb{C})$, with a non-Abelian identity component equal to the whole group $\operatorname{SL}(2, \mathbb{C})$.

Regarding exceptional values of energies given in (3.18): the condition that the first one is real gives $\beta\left(1+\mu^{3}+\beta^{2}(1+\mu)\right)=0$, which further suggests the equality (3.22). Its only physical solution is $m_{1}=0$. The second expression in (3.18) is never real and the third one is real when (3.22) holds only.

Acknowledgments This work was partially supported by the EC Marie Curie Network for Initial Training Astronet-II, Grant Agreement No. 289240, and by the National Science Centre, Poland, under grant DEC2012/05/B/ST1/02165.

Open Access This article is distributed under the terms of the Creative Commons Attribution License which permits any use, distribution, and reproduction in any medium, provided the original author(s) and the source are credited.

\section{Appendices}

A. Coefficients of polynomial $P$ in Eq. (3.17)

Explicit form of coefficients $p_{i}$ in $r$ given by (3.17) are as follows.

$$
\begin{aligned}
p_{0}= & \left.-8 e(1+\mu), \quad p_{1}=-8(1+\mu)(1+4 e(-1+\mu)+\mu)\right], \\
p_{2}= & -8(1+\mu)\left(-4+4 \mu^{2}+e\left(6+\beta^{2}+2 \mu(-5+3 \mu)\right)\right), \\
p_{3}= & -48 e^{2} \beta^{4}-16 e\left(-1+\mu^{2}\right)\left(2+2 \beta^{2}+\mu(-3+2 \mu)\right)-(1+\mu)^{2}\left(11 \beta^{2}\right. \\
& +24(2+\mu(-3+2 \mu))), \\
p_{4}= & -4\left(48 e^{2} \beta^{4}(-1+\mu)+(-1+\mu)(1+\mu)^{2}\left(8+11 \beta^{2}-6 \mu+8 \mu^{2}\right)\right. \\
& \left.+2 e(1+\mu)\left(1+12 \beta^{4}+\beta^{2}(6+\mu(-7+6 \mu))+\mu(-5+\mu(5+(-5+\mu) \mu))\right)\right), \\
p_{5}= & -2\left(-4(1+(e-\mu) \mu)\left(-1+\mu^{4}\right)+3 \beta^{4}\left(9(1+\mu)^{2}+64 e\left(-1+\mu^{2}\right)\right.\right. \\
& \left.\left.+16 e^{2}(3+\mu(-8+3 \mu))\right)+\beta^{2}\left(16 e\left(-1+\mu^{4}\right)+3(1+\mu)^{2}(11+\mu(-13+11 \mu))\right)\right), \\
p_{6}= & -4 \beta^{2}\left(48 e^{2} \beta^{2}(\mu-1)(1+(\mu-5) \mu)+(\mu-1)(1+\mu)^{2}\left(11+54 \beta^{2}\right.\right. \\
& +\mu(3+11 \mu))+2 e(1+\mu)\left(1+3 \beta^{2}(24+\mu(-59+24 \mu))\right. \\
& +\mu(-1+\mu(-5+(\mu-1) \mu)))), \\
p_{7}= & -\beta^{2}\left(11+3 \beta^{4}(1+\mu)^{2}+12 \beta^{2}\left(4 e\left(-8+33 \mu-33 \mu^{3}+8 \mu^{4}\right)+(1+\mu)^{2}(27\right.\right. \\
& \left.+\mu(-59+27 \mu))+4 e^{2}(1+(-2+\mu) \mu(8+(-14+\mu) \mu))\right)+\mu(32+\mu(-42 \mu \\
& \left.\left.\left.+\mu^{3}(32+11 \mu)-32 e\left(-1+\mu^{2}\right)\right)\right)\right), \\
p_{8}= & -4 \beta^{2}\left(3 \beta^{4}(-1+\mu)(1+\mu)^{2}-2(1+(e-\mu) \mu)\left(\mu+\mu^{4}\right)\right. \\
& +2 \beta^{2}\left(-24 e^{2}(-1+\mu) \mu(1+(-5+\mu) \mu)+27(-1+\mu)(1+\mu)^{2}(1+(-3+\mu) \mu)\right.
\end{aligned}
$$




$$
\begin{aligned}
& \left.\left.+2 e(1+\mu)\left(6+\mu\left(-76+\mu\left(157-76 \mu+6 \mu^{2}\right)\right)\right)\right)\right), \\
p_{9}= & -2 \beta^{4}\left(27+3 \beta^{2}(1+\mu)^{2}(3+\mu(-5+3 \mu))+\mu\left(-176-140 e\left(-1+4 \mu-4 \mu^{3}\right.\right.\right. \\
& \left.\left.\left.+\mu^{4}\right)+48 e^{2} \mu(3+\mu(-8+3 \mu))+\mu(4+\mu(414+\mu(4+\mu(-176+27 \mu))))\right)\right), \\
p_{10}= & -4 \beta^{4}\left(3 \beta^{2}\left(-1+\mu^{2}-\mu^{3}+\mu^{5}\right)-2 \mu(1+(e-\mu) \mu)(-11+\mu(24\right. \\
& +24 e(\mu-1)+(24-11 \mu) \mu))), \\
p_{11}= & -3 \beta^{4}\left(16 \mu^{2}(1+(e-\mu) \mu)^{2}+\beta^{2}\left(1+\mu^{3}\right)^{2}\right) .
\end{aligned}
$$

\section{B. Second-order differential equation in reduced form with rational coefficients and its differential Galois group}

Consider the second-order linear differential equation in reduced form with rational coefficient

$$
w^{\prime \prime}=r(z) w, \quad r(z) \in \mathbb{C}(z) .
$$

A point $z=c \in \mathbb{C}$ is a singular point of this equation if it is a pole of $r(z)$. A singular point is a regular point if at this point $(z-c)^{2} r(z)$ is holomorphic.

Assume that $c$ is a regular point and look for local solutions of this equation in a neighbourhood of this point. For simplicity, assume that $c=0$. Look for a solution of the following form (see e.g. Whittaker and Watson 1935),

$$
w=z^{\alpha} f(z), \quad f(z)=1+\sum_{i=1}^{\infty} f_{i} z^{i}
$$

where $\alpha, f_{i}$ for $i \in \mathbb{N}$ are constants to be determined. Substituting in (B.1) yields

$$
z^{\alpha-2}\left[\alpha(\alpha-1)+\sum_{i=1}^{\infty}(\alpha+i)(\alpha+i-1) f_{i} z^{i}\right]-r(z) z^{\alpha}\left(1+\sum_{i=1}^{\infty} f_{i} z^{i}\right)=0 .
$$

Multiplying by $z^{2}$, apply Taylor's formula for the analytic function $z^{2} r(z)$

$$
z^{2} r(z)=r_{0}+\sum_{i=1}^{\infty} r_{i} z^{i}
$$

and equating to zero the coefficients of successive powers of $t$, obtain the sequence of equations determining unknown coefficients in series (B.2). The first of these equations, called the indicial equation and determining $\alpha$, has the following form

$$
\alpha(\alpha-1)-r_{0}=0
$$

From the remaining equations $f_{i}$ is evaluated.

$$
f_{1}=\frac{r_{1}}{2 \alpha}, \quad f_{i}=\frac{1}{i(i+2 \alpha-1)}\left(\sum_{j=1}^{i-1} r_{j} f_{i-j}+r_{i}\right), \quad \text { for } \quad i \geq 2 .
$$


Let $\alpha_{1}$ and $\alpha_{2}$ be the roots of the indical equation. If the difference $s=\alpha_{1}-\alpha_{2}$ is not an integer, then Eq. (B.1) has two local solutions of the form

$$
w_{1}=z^{\alpha_{1}}\left(1+\sum_{i=1}^{\infty} f_{i} z^{i}\right), \quad w_{2}=z^{\alpha_{2}}\left(1+\sum_{i=1}^{\infty} f_{i}^{\prime} z^{i}\right) .
$$

In the case where $s$ is a positive integer or zero, the series $w_{2}$ may not exist or coincide with $w_{1}$, and a second independent solution must be constructed (Whittaker and Watson 1935) as

$$
w_{2}(z)=w_{1}(z) \int^{z} \frac{\mathrm{d} x}{w_{1}(x)^{2}}=w_{1}(z) \int^{z} z^{-2 \alpha_{1}} \frac{\mathrm{d} x}{f(x)^{2}}=w_{1}(z) \int^{z} z^{-s-1} \frac{\mathrm{d} x}{f(x)^{2}} .
$$

In the last equality the relation $2 \alpha_{1}=s+1$ is applied.

Denoting

$$
\frac{1}{f(z)^{2}}=1+\sum_{i=1}^{\infty} g_{i} z^{i}
$$

the second solution is written as

$$
w_{2}(z)=w_{1}(z) g_{s} \ln z+z^{\alpha_{2}}\left(-\frac{1}{s}+\sum_{i=1}^{\infty} h_{i} t^{i}\right),
$$

where $h_{i}$ are constants.

Coefficients $g_{i}$ are given by

$$
g_{1}=-2 f_{1}, \quad g_{2}=-2 f_{2}-2 f_{1} g_{1}-f_{1}^{2}=-2 f_{2}+3 f_{1}^{2},
$$

and

$$
g_{i}=-2 f_{i}-\sum_{j=1}^{i-1}\left(2 g_{i-j}+f_{i-j}\right) f_{j}-\sum_{j=1}^{i-2} f_{j} \sum_{k=1}^{i-j-1} f_{k} g_{i-j-k},
$$

for $i \geq 3$, while coefficients $h_{i}$ are defined as follows

$$
h_{i}= \begin{cases}-\frac{f_{1}}{s}-\frac{g_{1}}{s-1} & \text { for } i=1, \\ -\frac{f_{i}}{s}-\frac{g_{i}}{s-i}-\sum_{j=1}^{i-1} \frac{g_{j}}{s-j} f_{i-j} & \text { for } \quad 1<i<s, \\ -\frac{f_{s}}{s} & \text { for } i=s, \\ -\frac{f_{s+1}}{s}+g_{s+1}-\sum_{j=1}^{s-1} \frac{g_{j}}{s-j} f_{s+1-j} & \text { for } i=s+1, \\ -\frac{f_{i}}{s}+\frac{g_{i}}{i-s}-\sum_{j=1}^{s-1} \frac{g_{j}}{s-j} f_{i-j}+\sum_{j=s+1}^{i-1} \frac{g_{j}}{j-s} f_{i-j} & \text { for } i \geq s+2 .\end{cases}
$$

The differential Galois group of Eq. (B.1) is a subgroup of $\operatorname{SL}(2, \mathbb{C})$. Classification of these subgroups is well known. The following lemma shows that the differential Galois group determines the form of solutions (see e.g. Kovacic 1986; Morales Ruiz 1999).

Lemma B.1 Let $\mathcal{G}$ be the differential Galois group of equation (3.16). Then one of four cases may occur. 
Case I $\mathcal{G}$ is conjugate to a subgroup of the triangular group,

$$
\mathcal{T}=\left\{\left[\begin{array}{ll}
a & b \\
0 & a^{-1}
\end{array}\right] \mid a \in \mathbb{C}^{*}, b \in \mathbb{C}\right\} ;
$$

in this case equation (3.16) has an exponential solution of the form $y=P \exp \int \omega$, where $P \in \mathbb{C}[z]$ and $\omega \in \mathbb{C}(z)$.

Case II $\mathcal{G}$ is conjugate to a subgroup of

$$
\mathcal{D}^{\dagger}=\left\{\left[\begin{array}{ll}
c & 0 \\
0 & c^{-1}
\end{array}\right] \mid c \in \mathbb{C}^{*}\right\} \cup\left\{\left[\begin{array}{ll}
0 & c \\
-c^{-1} & 0
\end{array}\right] \mid c \in \mathbb{C}^{*}\right\} ;
$$

in this case equation (3.16) has a solution of the form $y=\exp \int \omega$, where $\omega$ is algebraic over $\mathbb{C}(z)$ of degree 2 ,

Case III $\mathcal{G}$ is primitive and finite; in this case all solutions of Eq. (3.16) are algebraic, thus $y=\exp \int \omega$, where $\omega$ belongs to an algebraic extension of $\mathbb{C}(z)$ of degree $n=4,6$ or 12.

Case IV $\mathcal{G}=\mathrm{SL}(2, \mathbb{C})$ and Eq. (3.16) has no Liouvillian solution.

Kovacic (1986) formulated the necessary conditions for the respective cases from Lemma B.1 to hold.

Write $r(z) \in \mathbb{C}(z)$ in the form

$$
r(z)=\frac{s(z)}{t(z)}, \quad s(z), t(z) \in \mathbb{C}[z],
$$

where $s(z)$ and $t(z)$ are relatively prime polynomials and $t(z)$ is monic. The roots of $t(z)$ are poles of $r(z)$. Denote $\Sigma^{\prime}:=\{c \in \mathbb{C} \mid t(c)=0\}$ and $\Sigma:=\Sigma^{\prime} \cup\{\infty\}$. The order $\operatorname{ord}(c)$ of $c \in \Sigma^{\prime}$ is equal to the multiplicity of $c$ as a root of $t(z)$, the order of infinity is defined by

$$
\operatorname{ord}(\infty):=\operatorname{deg} t-\operatorname{deg} s .
$$

Lemma B.2 The necessary conditions for the respective cases in Lemma B.1 are the following.

Case I. Every pole of $r$ must have even order or else have order 1. The order of $r$ at $\infty$ must be even or else be greater than 2 .

Case II. $r$ must have at least one pole that either has odd order greater than 2 or else has order 2.

Case III. The order of a pole of $r$ cannot exceed 2 and the order of $r$ at $\infty$ must be at least 2. If the partial-fraction expansion of $r$ is

$$
r(z)=\sum_{i} \frac{a_{i}}{\left(z-c_{i}\right)^{2}}+\sum_{j} \frac{b_{j}}{z-d_{j}},
$$

then $\Delta_{i}=\sqrt{1+4 a_{i}} \in \mathbb{Q}$, for each $i, \sum_{j} b_{j}=0$ and if

$$
g=\sum_{i} a_{i}+\sum_{j} b_{j} d_{j}
$$

then $\sqrt{1+4 g} \in \mathbb{Q}$. 


\section{References}

Arribas, M., Elipe, A., Riaguas, A.: Non-integrability of anisotropic quasi-homogeneous Hamiltonian systems. Mech. Res. Commun. 30, 209-216 (2003)

Boucher, D.: Non complere integrability of a satellite in circular orbit. Portugal. Math. 63(1), 69-89 (2006)

Boucher, D. and Weil, J.-A.: Application of J.-J. Morales and J.-P. Ramis' theorem to test the non-complete integrability of the planar three-body problem. Fauvet, F. (ed.) et al., From combinatorics to dynamical systems. Journées de calcul formel en l'honneur de Jean Thomann, Marseille, France, March 22-23, 2002. Berlin: de Gruyter. IRMA Lect. Math. Theor. Phys. 3, 163-177 (2003)

Burov, A., Kosenko, I., Troger, H.: On periodic motions of an orbital dumbbell-shaped body with a cabinelevator. Mech. Solids 47(3), 269-284 (2012)

Celletti, A.: Stability and Chaos in Celestial Mechanics. Springer Science+Business Media, New York (2010)

Celletti, A., Sidorenko, V.: Some properties of the dumbbell satellite attitude dynamics. Celest. Mech. Dyn. Astron. 101, 105-126 (2008)

Combot, T.: Non-integrability of the equal mass n-body problem with non-zero angular momentum. Celest. Mech. Dyn. Astron. 114, 319-340 (2012)

Combot, T.: A note on algebraic potentials and Morales-Ramis theory. Celes. Mech. Dyn. Astron. 115, 397-404 (2013)

Goździewski, K., Maciejewski, A.J.: Unrestricted planar of a symmetric body and a point mass. Triangular libration points and their stability. Celest. Mech. Dyn. Astron. 75, 251-285 (1999)

Guirao, J.L.G., Vera, J.A., Wade, B.A.: On the periodic solutions of a rigid dumbbell satellite in a circular orbit. Astrophys. Space Sci. 346, 437-442 (2013)

Khan, N., Goel, N.: Chaotic motion in problem of dumbell satellite. Int. J. Contemp. Math. Sci. 6(7), 299-307 (2011)

Kovacic, J.J.: An algorithm for solving second order linear homogeneous differential equations. J. Symb. Comput. 2(1), 3-43 (1986)

Maciejewski, A.J., Przybylska, M.: Non-integrability of ABC flow. Phys. Lett. A 303(4), 265-272 (2002)

Maciejewski, A.J., Przybylska, M.: Non-integrability of restricted two-body problems in constant curvature spaces. Regul. Chaotic Dyn. 8(4), 413-430 (2003a)

Maciejewski, A.J., Przybylska, M.: Non-integrability of the problem of a rigid satellite in gravitational and magnetic fields. Celest. Mech. Dyn. Astron. 87(4), 317-351 (2003b)

Maciejewski, A.J., Przybylska, M.: Non-integrability of the generalized two fixed centres problem. Celest. Mech. Dyn. Astron. 89(2), 145-164 (2004)

Maciejewski, A.J., Przybylska, M.: Non-integrability of three body problem. Celest. Mech. Dyn. Astron. 110(1), 17-30 (2011)

Morales Ruiz, J.J.: Differential Galois Theory and Non-Integrability of Hamiltonian systems, volume 179 of Progress in Mathematics. Birkhäuser Verlag, Basel (1999)

Morales-Ruiz, J.J., Simó, C., Simon, S.: Algebraic proof of the non-integrability of Hill's problem. Ergod. Theory Dyn. Syst. 25(4), 1237-1256 (2005)

Okunev, Y.M.: Possible motions of a long dumbbell in a central force field. Cosm. Res. 7, 578 (1969)

Povenmire, H.: Dumbbell shaped objects in the solar system. Meteor. Planet. Sci. Suppl. 37, 119 (2002)

Press, W. H., Teukolsky, S. A., Vetterling, W. T., and Flannery, B. P.: Numerical Recipes in C. The Art of Scientific Computing. Cambridge University Press, New York, second edition (1992)

Sanyal, A.K., Shen, J., McClamroch, N.H., Bloch, A.M.: Stability and stabilization of relative equilibria of dumbbell bodies in central gravity. J. Guid. Control Dyn. 28, 833-842 (2005)

Schechter, H.: Dumbbell librations in elliptic orbits. AIAA J 2, 1000-1003 (1964)

Simon, S.: On the meromorphic non-integrability of some problems in celestial mechanics. PhD thesis, Universitat de Barcelona, Spain (2007)

Tosel, E.J.: Un rèsultat de non-intégrabilité pour le potentiel en $1 / r^{2}$. C. R. Acad. Sci. Paris Série I 327, 387-392 (1998)

Tosel, E. J.: Non-intégrabilité algebrique et méromorphe de problémes de N corps. PhD thesis, University of Paris VI, Paris (1999)

Tsygvintsev, A.: The meromorphic non-integrability of the three-body problem. J. Reine Angew. Math 537, 127-149 (2001)

Tsygvintsev, A.V.: Non-existence of new meromorphic first integrals in the planar three-body problem. Celest. Mech. Dyn. Astron. 86(3), 237-247 (2003)

Tsygvintsev, A.V.: On some exceptional cases in the integrability of the three-body problem. Celest. Mech. Dyn. Astron. 99(1), 23-29 (2007) 
Vera, J.A.: On the periodic solutions of a rigid dumbbell satellite placed at of the restricted three body problem. Int. J. Non Linear Mech. 51, 152-156 (2013)

Whittaker, E.T., Watson, G.N.: A Course of Modern Analysis. Cambridge University Press, London (1935) 